\title{
Why Do the Relativistic Masses and Momenta of Faster-than-Light Particles Decrease as their Speeds Increase?
}

\author{
Judit X. MADARÁSZ ${ }^{\dagger}$, Mike STANNETT ${ }^{\ddagger}$ and Gergely SZÉKELY ${ }^{\dagger}$ \\ † Alfréd Rényi Institute of Mathematics, Hungarian Academy of Sciences, \\ P.O. Box 127, Budapest 1364, Hungary \\ E-mail: madarasz.judit@renyi.mta.hu,szekely.gergely@renyi.mta.hu \\ $\ddagger$ University of Sheffield, Department of Computer Science, \\ 211 Portobello, Sheffield S1 4DP, United Kingdom \\ E-mail: m.stannett@sheffield.ac.uk
}

Received September 17, 2013, in final form January 07, 2014; Published online January 11, 2014 http://dx.doi.org/10.3842/SIGMA.2014.005

\begin{abstract}
It has recently been shown within a formal axiomatic framework using a definition of four-momentum based on the Stückelberg-Feynman-Sudarshan-Recami "switching principle" that Einstein's relativistic dynamics is logically consistent with the existence of interacting faster-than-light inertial particles. Our results here show, using only basic natural assumptions on dynamics, that this definition is the only possible way to get a consistent theory of such particles moving within the geometry of Minkowskian spacetime. We present a strictly formal proof from a streamlined axiom system that given any slow or fast inertial particle, all inertial observers agree on the value of $\mathrm{m} \cdot \sqrt{\left|1-v^{2}\right|}$, where $\mathrm{m}$ is the particle's relativistic mass and $v$ its speed. This confirms formally the widely held belief that the relativistic mass and momentum of a positive-mass faster-than-light particle must decrease as its speed increases.
\end{abstract}

Key words: special relativity; dynamics; faster-than-light particles; superluminal motion; tachyons; axiomatic method; first-order logic

2010 Mathematics Subject Classification: 70A05; 03B30; 83A05

\section{Introduction}

Following the introduction of Einstein's special theory of relativity in 1905, it was generally believed that the existence of faster-than-light (FTL) particles would violate causality, and they could not therefore exist. Tolman's 1917 'anti-telephone' scenario [33] was followed by a series of papers describing causality violations involving FTL particles, leading over the last half-century to a host of related paradoxes and possible resolutions $[4,5,6,8,11,12,16,19,21,22,25,26$, $31,34,35]$.

Another issue associated with FTL particles concerns the nature of collisions. If one observer sees two particles (one of which is FTL) fusing to form a third, then another, fast enough but nonetheless slower-than-light, observer will see this fusion as the decay of one particle into two others (see Fig. 1) [21, 31]. Such ambiguities are not in themselves paradoxical; rather, they provide one more confirmation of the need to distinguish between physical laws and their visible manifestations in relativity theory [5].

Various authors have investigated how the relativistic mass of FTL particles ought to vary with speed, prompted in part by experimental evidence confirming the existence of FTL behaviours, e.g., in certain astronomical and quantum-mechanical situations (see [21] for a com- 


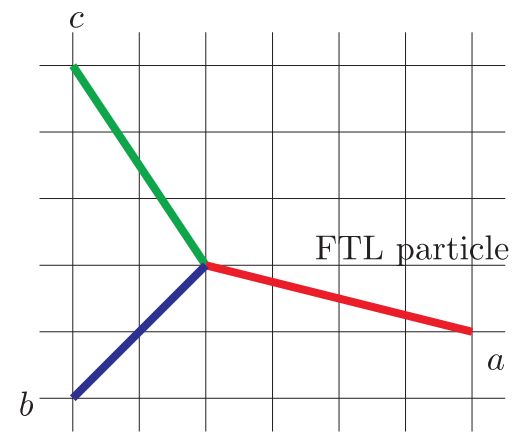

FUSION

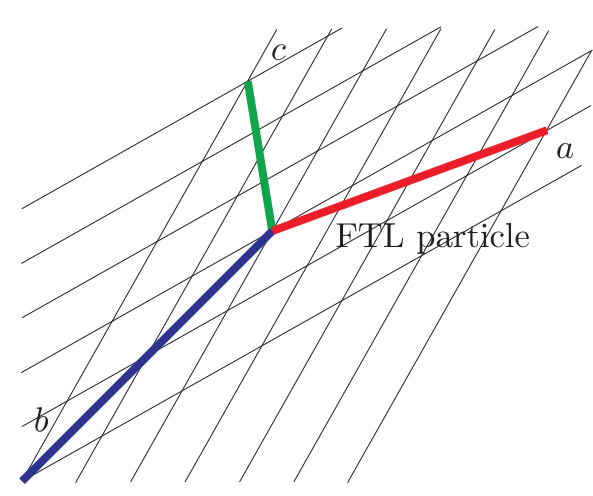

DECAY

Figure 1. If FTL particles are involved, what one person sees as a decay, another may see as fusion.

prehensive review). Sudarshan [31] considered the theory of tachyons, and noted - contrary to earlier assumptions in the literature - that their existence does not contradict any of the physical consequences of relativity theory, since these are based not on the 'limiting' nature of the speed of light, but its invariance. Even more strikingly, Recami [24] notes that "at least four different experimental sectors of physics seem to indicate the actual existence of Superluminal motions", two of which have been confirmed both theoretically and experimentally [23]. In particular, various experiments [14, 17, 20, 29] (cf. [18]) have confirmed the quantum-theoretical prediction [1] that the total time taken for a photon to 'tunnel' through an opaque barrier is independent of tunnel width, whence group velocities are necessarily superluminal for wide enough barriers.

The derivations presented in these earlier papers differ from ours in certain respects. For example, Recami and his colleagues follow Sudarshan [31] in assuming that positive-energy objects travelling backwards in time cannot exist, and that "any negative-energy particle $P$ travelling backwards in time can and must be described as its antiparticle $\bar{P}$, endowed with positive energy and motion forward in time", whereas we make no such blanket assumption. Recami [22] suggests that since we know nothing a priori about tachyons, "the safest way to formulate a theory for them is to try to generalize the ordinary theories ... by performing modifications as small as possible". Our approach is to remove unnecessary assumptions so as to generalise the standard theory to the point where tachyon dynamics can be investigated without further modification.

Our derivation is, accordingly, by strict formal proof from a sparse set of basic, simple, axioms that are already known to be consistent both with standard descriptions of special relativity and with the existence of FTL particles [15]. We show, moreover, how the same deductive reasoning can be applied to Newtonian as well as relativistic dynamics, by replacing just one of the underlying axioms. Our main theorem (Theorem 1) shows that

- if relativistic dynamics holds, then all inertial observers agree on the value they calculate for $\mathrm{m} \cdot \sqrt{\left|1-v^{2}\right|}$, where $\mathrm{m}$ is the relativistic mass of an inertial particle and $v$ is its speed, whether or not this is $\mathrm{FTL}^{1}$;

- if Newtonian dynamics holds, all observers agree on the particle's relativistic mass m.

A consequence of this result is confirmation that if an FTL inertial particle has positive relativistic mass, its relativistic mass and momentum must decrease as its speed increases, while for slow particles we get back the usual mass increase theorem in the relativistic case.

\footnotetext{
${ }^{1}$ For 'slow' particles, this invariant is the usual rest mass $\mathrm{m}_{0}=\mathrm{m} \cdot \sqrt{1-v^{2}}$. For FTL particles, our formula $\mathrm{m} \cdot \sqrt{v^{2}-1}$ agrees with that required by non-restricted special relativity (NRR) [21] (although, of course, we are working here not in NRR, but in a general axiomatic framework of dynamics).
} 
In [15] a model of relativistic dynamics is constructed in which there are interacting FTL inertial particles, by extending the definition of four-momentum for FTL particles using the Stückelberg-Feynman-Sudarshan-Recami "switching principle" [5, 9, 30]. Our results here therefore complement [15], because they show - using only basic natural assumptions on dynamics - that this definition is the only possible way to get a consistent theory of interacting FTL inertial particles.

\section{Informal formulation of our axioms and the main result}

We begin with an informal outline of our basic first-order axiom system, BA. This formalises the axioms used in standard approaches to special relativity theory from Einstein onwards. Informally paraphrased, these axioms are:

- AxEField Physical quantities satisfy certain basic algebraic properties of the real numbers.

- AxL The world-line of every inertial particle and inertial observer is a subset of a straight line, and contains at least two points.

- $\mathrm{AxW}$

The world-view transformation between any two observers is an affine transformation which takes the world-line of each body according to the first observer to the world-line of the same body according to the second observer.

- AxSelf-

Inertial observers are stationary (they do not move spatially) in their own coordinate systems.

Using BA, we can define the speeds and velocities of inertial observers and inertial particles according to any observer, as well as the linear momenta of inertial particles moving at finite relative speeds.

Our next axiom system, DYN (for dynamics), adds concepts relating to particle collisions. Intuitively, by a collision we mean a set of incoming and outgoing inertial particles for which the sums of the relativistic masses and linear momenta of the incoming particles coincide with those of the outgoing ones (Fig. 2), i.e. we build conservation of both relativistic mass and linear momentum into collisions from the outset. Inelastic collisions are defined as collisions in which there is only one outgoing particle ${ }^{2}$.

Informally, DYN consists of the basic assumptions in BA together with the following axioms (see Fig. 4):

- $\mathrm{AxColl} 3$

If one observer sees a 3-particle collision, all the others see it as well.

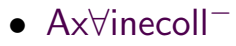

Given any two inertial particles $a$ and $b$ with given velocities and relativistic masses, we can find a (possibly different) pair of particles $a^{\prime}$ and $b^{\prime}$ which can collide inelastically, where the relativistic masses and velocities of $a^{\prime}$ and $b^{\prime}$ agree with those of $a$ and $b$ respectively.

- $\mathrm{AxSpd} \mathrm{d}^{-}$

If two observers agree on the speed of a 'slow' inertial particle, then they agree on its relativistic mass.

\footnotetext{
${ }^{2}$ As is well known, when two particles undergo a relativistic inelastic collision, kinetic energy is not conserved [10]. Nonetheless, the total energy of the system - and hence its combined relativistic mass - remains unchanged [7, § 4.3], [13, Example 3.2], [27, § 6.2].
} 

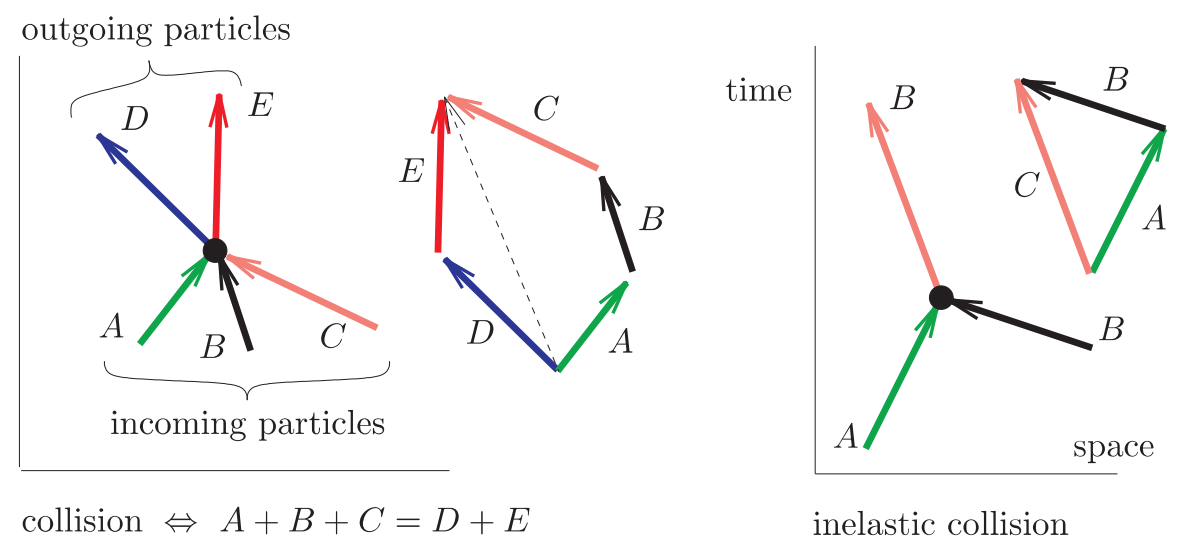

inelastic collision

Figure 2. Collision and inelastic collision of incoming and outgoing particles. Four-momentum is conserved in collisions.

Notice, however, that we make no assumptions concerning the relationship between relativistic mass and speed for FTL particles. Instead, this will emerge as a logical consequence of the axioms.

- AxMass

If the velocities and relativistic masses of two inertial particles coincide for one observer, then their relativistic masses also coincide for all other observers.

- AxThEx ${ }^{-}$

Given any observers $m$ and $h$, and any non-negative speed $v$ slower than that of $h$ as observed by $m$, we can assume the existence of a massive inertial particle with speed $v$ relative to $m$.

In relativistic terms, AxThEx- says that inertial particles with non-zero relativistic mass can be assumed to travel at any desired sub-light speed (because observer $h$ cannot travel faster than light in our framework), but the axiom is phrased so as to remain valid in Newtonian dynamics also. The difference between the relativistic and Newtonian systems is captured by

- AxPh, the light axiom

The speed of light is 1 for every observer;

- AxAbsSim, absolute simultaneity

If two events are simultaneous for one observer, then they are simultaneous for all observers.

In our theories, relativistic dynamics is captured by DYN+AxPh, while DYN+AxAbsSim is an axiom system for Newtonian dynamics [15].

Writing $\mathrm{m}_{k}(b)$ for the relativistic mass and $v_{k}(b)$ for the speed of particle $b$ according to observer $k$, the main result of this paper (Theorem 1) describes how particle mass (including that of FTL particles) varies with speed in both relativistic and Newtonian dynamics. For all inertial observers $k$ and $h$, and any inertial particle $b$ moving with a finite speed according to both of them, we have

- if relativistic dynamics (DYN+AxPh) holds, then

$$
\mathrm{m}_{k}(b) \cdot \sqrt{\left|1-v_{k}(b)^{2}\right|}=\mathrm{m}_{h}(b) \cdot \sqrt{\left|1-v_{h}(b)^{2}\right|} ;
$$

- if Newtonian dynamics (DYN+AxAbsSim) holds, then

$$
\mathrm{m}_{k}(b)=\mathrm{m}_{h}(b) .
$$


Equation (1) gives back the usual relativistic mass-increase theorem for slower than light particles, but predicts that both the relativistic mass and the momentum of an FTL particle with positive mass should decrease with speed.

It is worth noting that the axiom system $D Y N+A x P h$ is self-consistent because it is more general that the axiom system SRDyn of [15], which has been shown there to be logically consistent. Likewise, a similar construction to that used in [15] shows that the axiom system DYN+AxAbsSim is also self-consistent.

\section{Formalization}

We begin by formalising a number of basic concepts, including the axioms described above, and introduce some notation. For brevity we have adopted the familiar expositional style of formal mathematics, but it should be noted that everything can also be expressed in the pure first-order logic framework of, e.g., $[3,15]$.

\subsection{Quantities and bodies}

We use the algebraic structure $Q \equiv\langle Q,+, \cdot,<\rangle$ to represent quantities (the values used to measure relativistic masses, speed, momenta, etc.), where + (addition) and · (multiplication) are binary operations, and $<$ (less than) is a binary relation, on the set $Q$. As usual, we write $Q^{n}$ for the Cartesian product of $n$ copies of $Q$, and write $x_{i}$ for the $i^{\text {th }}$ component of $\bar{x}=\left(x_{1}, \ldots, x_{n}\right) \in Q^{n}$.

We write $B$ for the set of bodies, and pick out three types of body in particular: the sets IOb of inertial observers, Ip of inertial particles and $\mathrm{Ph}$ of photons are subsets of $B$. We do not assume a priori that these subsets are disjoint - the fact that, e.g., photons cannot be inertial observers in the relativistic case, arises instead as a theorem of the logic.

Given any inertial observers $k, h \in \mathrm{IOb}$ and body $b \in B$,

- $w \ell_{k}(b) \subseteq Q^{4}$ is the world-line of $b$ according to observer $k$;

- $\mathbf{w}_{k h}: Q^{4} \rightarrow Q^{4}$ is the world-view transformation between the world-views (coordinate systems) of $k$ and $h$;

- $\mathrm{m}_{k}(b) \in Q$ is the relativistic mass of $b$ according to $k$.

\subsection{Basic axioms}

Throughout the paper we assume a set BA of four basic axioms in our definitions and axioms without mentioning this explicitly. Formally, we have

$$
B A:=\left\{\text { AxEField }, A x L, A \times W, A \times S_{e l f}{ }^{-}\right\}
$$

where the axioms AxEField, AxL, AxW and $\mathrm{AxSelf}^{-}$are defined below.

\subsubsection{Notation}

Our notation is essentially standard. We write $\mathbb{R}$ for the field of real numbers and $:=$ for definitional equality, so that " $v:=e$ " indicates that the value $v$ is fully determined by evaluating an appropriate instantiation of the expression $e$. Given any function $f: X \rightarrow Y$ and $S \subseteq X$, the $f$-image of $S$ is given by $f[S]:=\{f(x): x \in S\}$. 


\subsubsection{Field axioms}

Einstein and his followers assumed implicitly that the structure $Q$ is the field $\mathbb{R}$ of real numbers, but in fact this imposes more logical structure than is necessary for our deductions. We therefore make our system more general by assuming for $Q$ only the more important algebraic properties of $\mathbb{R}$.

AxEField The quantity part $\langle Q,+, \cdot,<\rangle$ is assumed to be a Euclidean field, i.e. it is a linearly ordered field in the sense of abstract algebra, equipped with the usual field operations 0 , $1,-$ and / definable from addition and multiplication, and every non-negative element $x$ has a square root, i.e. $(\forall x>0)(\exists y)\left(x=y^{2}\right)$. It is easy to see that square roots can be assumed to be non-negative when they exist, and we define the $\sqrt{ }$ function accordingly.

For each $n=1,2,3, \ldots$, the set $Q^{n}$ is be assumed to carry the normal vector space structure. Regardless of $n$, we write $\overline{0}:=(0, \ldots, 0)$ for the origin. The Euclidean length of a vector $\bar{x}=\left(x_{1}, \ldots, x_{n}\right)$ is the non-negative quantity $|\bar{x}|=\sqrt{x_{1}^{2}+\cdots+x_{n}^{2}}$.

For simplicity, we shall make the standard assumption throughout this paper that $n=4$, so that spacetime has one temporal and three spatial dimensions. Given any $\bar{x}=\left(x_{1}, x_{2}, x_{3}, x_{4}\right) \in$ $Q^{4}$ we call $x_{1}$ its time component and $\left(x_{2}, x_{3}, x_{4}\right)$ its space component. If $\bar{x}$ has time component $t$ and space component $\bar{s}$, we occasionally abuse notation and write $\bar{x}=(t, \bar{s})$.

\subsubsection{World-lines}

We assume that the world-line of every inertial particle or inertial observer is a subset of a straight $\operatorname{line}^{3}$ containing at least 2 distinct points.

AxL For every $k \in \mathrm{IOb}$ and $b \in \mathrm{Ip} \cup \mathrm{IOb}$ there is a straight line $\ell$ of $Q^{4}$ such that $w \ell_{k}(b) \subseteq \ell$, and $w \ell_{k}(b)$ contains at least two points (elements).

\subsubsection{World-view transformation}

The world-view transformation between any two observers is an affine transformation (i.e. a linear transformation composed with a translation) which takes the world-line of each body according to the first observer to its world-line according to the second observer.

$\underline{\mathrm{AxW}} \mathbf{w}_{k h}$ is an affine transformation and $\mathbf{w}_{k h}\left[w \ell_{k}(b)\right]=w \ell_{h}(b)$ for every $k, h \in \mathrm{IOb}$ and $b \in B$.

\subsubsection{Self-coordinatisation}

Inertial observers consider themselves to be stationary in space (but not in time). This makes it easy to speak about the motion of inertial observers since it identifies observers with their time axes.

$\underline{\text { AxSelf }^{-}}$If $\bar{x} \in w \ell_{k}(k)$, then $\left(x_{2}, x_{3}, x_{4}\right)=(0,0,0)$.

\subsubsection{Auxiliary definitions}

The velocity $\mathbf{v}_{k}(b) \in Q^{3}$ and speed $v_{k}(b) \in Q$ of any inertial body $b \in \mathrm{Ip} \cup \mathrm{IOb}$ according to an inertial observer $k$ are defined by

$$
\mathbf{v}_{k}(b):=\left(x_{2}-y_{2}, x_{3}-y_{3}, x_{4}-y_{4}\right) /\left(x_{1}-y_{1}\right) \quad \text { and } \quad v_{k}(b):=\left|\mathbf{v}_{k}(b)\right|,
$$

\footnotetext{
${ }^{3}$ By a straight line we mean a set $\ell \subseteq Q^{4}$ for which there exist distinct $\bar{x}, \bar{y} \in Q^{4}$ with $\ell=\{\bar{x}+q \cdot(\bar{y}-\bar{x}):$ $q \in Q\}$.
} 
where $\bar{x}, \bar{y} \in w \ell_{k}(b)$ are any points for which $x_{1} \neq y_{1}$ (these are well defined concepts whenever such points exist, since they do not depend on the choice of $\bar{x}$ and $\bar{y}$ ).

If there are no such $\bar{x}$ and $\bar{y}$, then $\mathbf{v}_{k}(b)$ and $v_{k}(b)$ are undefined. Indeed, if there are distinct $\bar{x}, \bar{y} \in w \ell_{k}(b)$ with $x_{1}=y_{1}$, the worldline of $b$ is a subset of a horizontal straight line and its speed is, from $k$ 's viewpoint, 'infinite'. We therefore write $v_{k}(b)=\infty$ in this case, and $v_{k}(b)<\infty$ otherwise. We say that the speed of body $b$ is finite (infinite) according to observer $k$ provided $v_{k}(b)<\infty\left(v_{k}(b)=\infty\right)$, respectively.

\section{Axioms for dynamics}

Throughout this paper we write $\mathrm{m}_{k}(b)$ for the relativistic mass of particle $b$ according to observer $k$ - we assume here that $\mathrm{m}$ is a primitive construct and deduce (Theorem 1 ) how $\mathrm{m}_{k}(b)$ varies with the relative speed of $b$ relative to $k$.

We define the linear momentum $\mathbf{p}_{k}(b)$ of inertial particle $b$ according to inertial observer $k$ in the obvious way, viz. $\mathbf{p}_{k}(b)=\mathrm{m}_{k}(b) \cdot \mathbf{v}_{k}(b)$. Provided $b$ is travelling at finite speed relative to $k$, so that $\mathbf{p}_{k}(b)$ is defined, the associated four-momentum $\mathrm{P}_{k}(b)$ is the vector in $Q^{4}$ whose time component is the relativistic mass, and whose space component is the linear momentum it is not difficult to prove that $\mathrm{P}_{k}(b)$ is parallel to the world-line of $b$ (Fig. 3)

$$
\mathrm{P}_{k}(b)= \begin{cases}\left(\mathrm{m}_{k}(b), \mathbf{p}_{k}(b)\right) & \text { if } v_{k}(b)<\infty \\ \text { undefined } & \text { otherwise }\end{cases}
$$

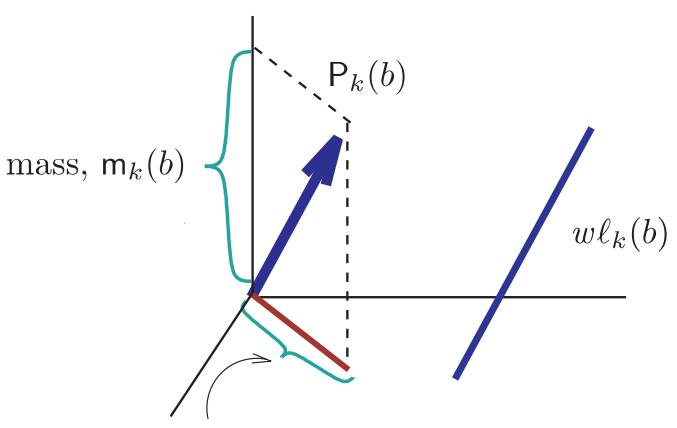

linear momentum, $\mathbf{p}_{k}(b)$

Figure 3. The time component of four-momentum is relativistic mass, and its space component is linear momentum. Each inertial observer $k$ considers $b$ 's four-momentum to be parallel to its world-line.

\subsection{Particles involved in a collision}

We say that an inertial particle $b$ is incoming at some coordinate point $\bar{x}$, according to inertial observer $k$, provided $\bar{x} \in w \ell_{k}(b)$ and $x_{1}$ is an upper bound for the time coordinates of points on $w \ell_{k}(b)$ and the speed of $b$ is finite, i.e. if $\bar{y} \in w \ell_{k}(b)$ and $\bar{y} \neq \bar{x}$ then $y_{1}<x_{1}$. We write $\operatorname{in}_{k}(b)$ to mean that there is some point $\bar{x}$ at which $b$ is incoming according to $k$, and $\operatorname{in}_{k}(b) @ \bar{x}$ if we wish to highlight some such $\bar{x}$ explicitly. Outgoing particles, out ${ }_{k}(b)$ and $\operatorname{out}_{k}(b) @ \bar{x}$ are defined analogously.

A collection $b_{1}, \ldots, b_{n}$ of inertial particles form a collision according to observer $k$ provided

- there is a point $\bar{x}$ at which they are all either incoming or outgoing with finite speed according to $k$; and 
- relativistic mass and linear momentum are both conserved at $\bar{x}$ according to $k$ (Fig. 2), i.e. allowing $b_{i}$ to range over the particles, we have

$$
\sum_{\left\{b_{i}: \mathrm{in}_{k}\left(b_{i}\right) @ \bar{x}\right\}} \mathrm{P}_{k}\left(b_{i}\right)=\sum_{\left\{b_{i}: \text { out }_{k}\left(b_{i}\right) @ \bar{x}\right\}} \mathrm{P}_{k}\left(b_{i}\right) .
$$

We are particularly interested below in collisions involving three particles, and write $\mathrm{coll}_{k}(a b c)$ to mean that $a, b, c$ form a collision according to $k$.

\subsubsection{Collision axioms}

For each natural number $n$ we introduce an axiom saying that the existence of any $n$-body collision is observer-independent. Thus, conservations of relativistic mass, linear momentum and four-momentum do not depend on the inertial observer.

AxColl $\mathrm{n}$ If inertial particles $b_{1}, \ldots, b_{n}$ form a collision for one inertial observer, they also form a collision for every other inertial observer who considers them all to have finite speed (see Fig. 4).

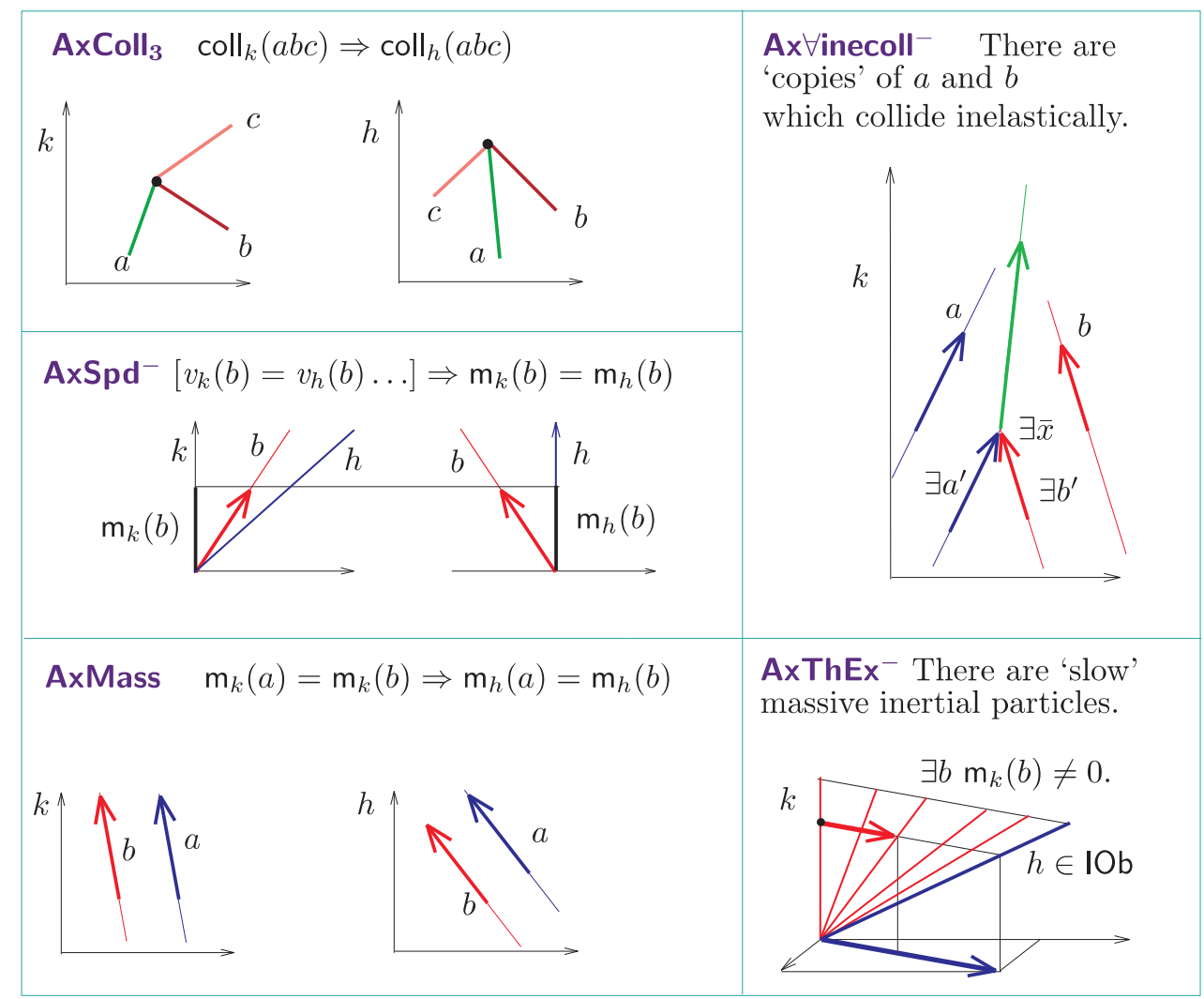

Figure 4. The dynamical axioms in DYN.

\subsubsection{Inelastic collisions}

We say that inertial particles $a$ and $b$ collide inelastically according to observer $k$ provided there exist some inertial particle $c$ such that $a, b, c$ form a collision, where $a$ and $b$ are incoming and $c$ is outgoing, i.e.

$$
\exists c\left[\operatorname{coll}_{k}(a b c) \wedge \operatorname{in}_{k}(a) \wedge \operatorname{in}_{k}(b) \wedge \operatorname{out}_{k}(c)\right] .
$$


The following axiom is slightly subtle. In general, an observer would not expect two arbitrarily selected particles $a$ and $b$ to be following worldlines that necessarily meet. We can, however, find paths parallel to those followed by $a$ and $b$ (i.e. followed by particles with the same fourmomenta), which meet at some point. The axiom states this formally, and says that we can form an inelastic collision of these parallel-moving particles at $\bar{x}$, provided they have 'sensible' relativistic masses and speeds according to the observer in question. In other words, this axiom says that all 'sensible' collisions are realisable.

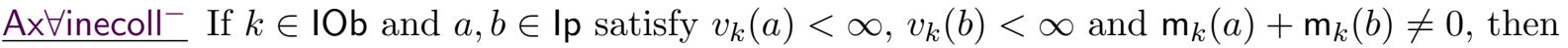
there exist $a^{\prime}, b^{\prime} \in \mathrm{Ip}$ with $\mathrm{P}_{k}\left(a^{\prime}\right)=\mathrm{P}_{k}(a)$ and $\mathrm{P}_{k}\left(b^{\prime}\right)=\mathrm{P}_{k}(b)$, such that $a^{\prime}$ and $b^{\prime}$ collide inelastically.

We typically use these collisions to determine relationships between the four-momenta of $a$ and $b$, but of course it is actually $a^{\prime}$ and $b^{\prime}$ that take part in the collision. Nonetheless our deductions remain valid, because $a$ and $a^{\prime}$ have the same four-momentum, as do $b$ and $b^{\prime}$, and these equalities continue to hold in every other observer's worldview by AxMass below.

\subsubsection{Relativistic mass}

Given $\bar{u}, \bar{v} \in Q^{3}$, we write $\bar{u} \preceq \bar{v}$ to mean that $\bar{u}$ and $\bar{v}$ point in the same direction, and the length of $\bar{u}$ is less than or equal to the length of $\bar{v}$, i.e.

$$
\bar{u} \preceq \bar{v} \quad \stackrel{\text { def }}{\Longleftrightarrow} \exists \lambda(0 \leq \lambda \leq 1 \wedge \bar{u}=\lambda \cdot \bar{v}) .
$$

The following axioms concern the relativistic masses of slow particles, and are expressed in a way that makes them equally meaningful for both relativistic and Newtonian dynamics. Since inertial observers travel slower than light in the relativistic setting, but can achieve any sublight speed we wish, we can define a particle to be 'slow' provided there is some observer who appears to be travelling faster than it. That is, observer $k$ considers body $b$ to be 'slow' provided there is some other observer $h$ for which $\mathbf{v}_{k}(b) \preceq \mathbf{v}_{k}(h)$.

Our next axiom asserts that whenever two observers agree on the speed of a slow inertial particle, then they also agree on its relativistic mass. That is, suppose observer $k$ considers inertial particle $b$ and observer $h$ to be moving in the same direction, with $h$ moving faster than $b$. If $h$ and $k$ see $b$ to be moving at the same speed, they will also agree on its relativistic mass (Fig. 4).

$\underline{\mathrm{AxSpd}^{-}}$If $\mathbf{v}_{k}(b) \preceq \mathbf{v}_{k}(h)$ and $v_{k}(b)=v_{h}(b)$, then $\mathrm{m}_{k}(b)=\mathrm{m}_{h}(b)$, for all $k, h \in$ IOb and $b \in \mathrm{Ip}$.

It is important in axiom $\mathrm{AxSpd}^{-}$that particle $b$ is slow, because in special relativity, if observers $k$ and $h$ move with respect to one another and $b$ is moving with the speed of light in the same spatial direction as $h$ according to $k$, then the speed of $b$ is the same for $k$ and $h$ but its relativistic mass is different. In both relativistic and Newtonian axiom systems, we can prove that no observer can travel with infinite speed, and moreover in the relativistic axiom system every observer travels with slower than light speed, see Corollary 1.

We also assume that if the relativistic masses and velocities of two inertial particles coincide for one inertial observer, then their relativistic masses also coincide for all other inertial observers.

AxMass If $\mathrm{P}_{k}(a)=\mathrm{P}_{k}(b)$, then $\mathrm{m}_{h}(a)=\mathrm{m}_{h}(b)$ for all $k, h \in \mathrm{IOb}$ and $a, b \in \mathrm{Ip}$.

Finally, we assume the existence of 'slow' inertial particles with nonzero relativistic masses. That is, given any inertial observer and any 'slow' velocity $\bar{v}$, there is an inertial particle with nonzero relativistic mass that moves with velocity $\bar{v}$.

AxThEx ${ }^{-}$For all $k, h \in \mathrm{IOb}$ and $\bar{v} \preceq \mathbf{v}_{k}(h)$ there is $b \in \mathrm{Ip}$ satisfying $\mathbf{v}_{k}(b)=\bar{v}$ and $\mathrm{m}_{k}(b) \neq 0$. 


\section{$\mathrm{AxPh}$}

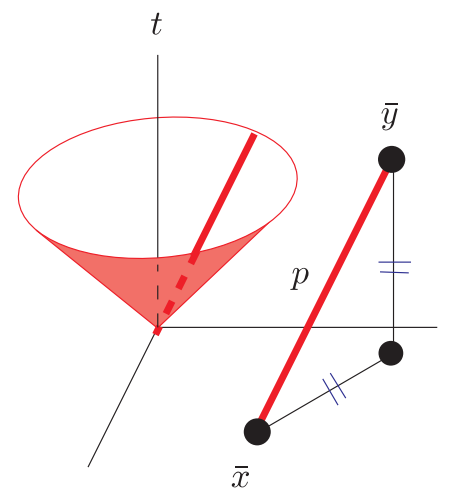

AxAbsSim

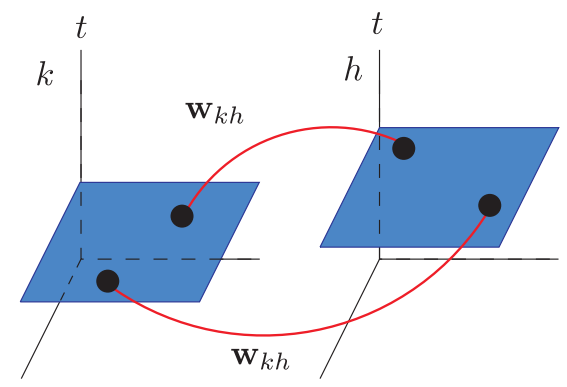

Figure 5. Illustration for the photon axiom and for the absolute simultaneity axiom.

\subsubsection{Shared axiom system for dynamics}

We can now define the axiom system DYN for dynamics, which is common to both the relativistic and Newtonian settings. It comprises the basic assumptions, together with the axioms introduced above, viz.

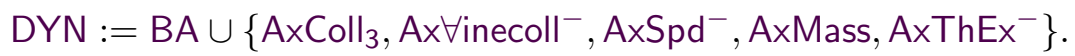

\subsubsection{Differentiating between relativistic and Newtonian dynamics}

Finally, we define the axioms that allow us to distinguish between the two systems (Fig. 5).

The photon axiom, $\mathrm{AxPh}$, says that each inertial observer considers the speed of light to be 1 everywhere and in every direction (in particular, therefore, it is finite, whence this axiom characterises relativistic dynamics). Moreover, it is always possible to send out such light signals, i.e. two points lie on a photon's worldline if and only if the slope of the line joining them is 1.

$\underline{\mathrm{AxPh}}$ For every inertial observer $k \in \mathrm{IOb}$,

$$
(\exists p \in \mathrm{Ph})\left(\bar{x}, \bar{y} \in w \ell_{k}(p)\right) \quad \Leftrightarrow \quad\left(x_{2}-y_{2}\right)^{2}+\left(x_{3}-y_{3}\right)^{2}+\left(x_{4}-y_{4}\right)^{2}=\left(x_{1}-y_{1}\right)^{2} .
$$

In contrast, the absolute simultaneity axiom, AxAbsSim, which characterises Newtonian dynamics, says that whenever two events are simultaneous for one observer then they are simultaneous for every observer.

AxAbsSim If $\mathbf{w}_{k h}(\bar{x})=\bar{x}^{\prime}$ and $\mathbf{w}_{k h}(\bar{y})=\bar{y}^{\prime}$, then $x_{1}=y_{1} \Leftrightarrow x_{1}^{\prime}=y_{1}^{\prime}$ for every $k, h \in \mathrm{IOb}$.

The axiom system DYN $\cup\{\mathrm{AxPh}\}$ is our axiom system for relativistic dynamics, and axiom system DYN $\cup\{$ AxAbsSim $\}$ is our axiom system for Newtonian dynamics.

\section{The main theorem and its proof}

We can now state and prove our main theorem. Our central claim, concerning the way that relativistic masses transform as speed increases, then follows as an easy corollary.

Theorem 1. Assume axiom system DYN. Let $b$ be an inertial particle and let $k, h$ be inertial observers for which the speed of $b$ is finite. Then

(a) $\mathrm{m}_{k}(b) \cdot \sqrt{\left|1-v_{k}(b)^{2}\right|}=\mathrm{m}_{h}(b) \cdot \sqrt{\left|1-v_{h}(b)^{2}\right|}$ if $\mathrm{AxPh}$ holds;

(b) $\mathrm{m}_{k}(b)=\mathrm{m}_{h}(b)$ if AxAbsSim holds. 


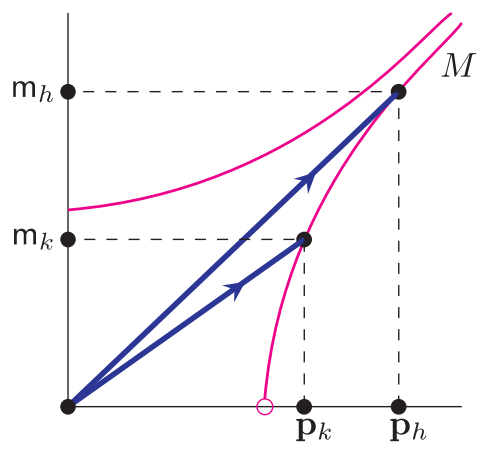

Figure 6. A spacetime diagram showing the relativistic mass $m$ and linear momentum $\mathbf{p}$ of a positivemass FTL body $b$ as seen by two observers $k$ and $h$ in the relativistic setting. The arrows represent the four-momenta of $b$ as seen by each observer, and the continuous lines show the Minkowski circle on which their tips are constrained to lie.

To see how positive FTL mass and momentum change in the relativistic setting depending on the particle's speed relative to an inertial observer, let us assume that $b$ is an FTL particle relative to $k$, and that $v_{h}(b)<v_{k}(b)$. It is known [28] that the axioms assumed here prohibit inertial observers (though not FTL inertial particles in general) from travelling FTL with respect to one another, so we can also assume that $b$ is travelling FTL relative to $h$. Since both observers consider $v(b)$ to be greater than 1 , the theorem tells us that

$$
\mathrm{m}_{k}(b)=\mathrm{m}_{h}(b) \cdot \sqrt{\frac{v_{h}(b)^{2}-1}{v_{k}(b)^{2}-1}}<\mathrm{m}_{h}(b),
$$

so that relativistic mass is considered to be lower by the observer who is moving faster relative to the particle. Similarly, the observers' momentum measurements satisfy

$$
\left|\mathbf{p}_{k}(b)\right|=\mathrm{m}_{k}(b) \cdot v_{k}(b)=\mathrm{m}_{h}(b) \cdot \sqrt{\frac{v_{h}(b)^{2}-1}{v_{k}(b)^{2}-1}} \cdot v_{k}(b) .
$$

A simple rearrangement of terms shows that

$$
v_{k}(b)>v_{h}(b) \Rightarrow \sqrt{\frac{v_{k}(b)^{2} \cdot\left(v_{h}(b)^{2}-1\right)}{v_{k}(b)^{2}-1}}<v_{h}(b),
$$

and hence

$$
\left|\mathbf{p}_{k}(b)\right|=\mathbf{m}_{k}(b) \cdot v_{k}(b)<\mathrm{m}_{h}(b) \cdot v_{h}(b)=\left|\mathbf{p}_{h}(b)\right|,
$$

so that momentum also appears to drop as the particle's speed increases.

We can also interpret this result as shown in Fig. 6. Notice that $\mathrm{m}_{k}(b) \cdot \sqrt{\left|1-v_{k}(b)^{2}\right|}$ is essentially the Minkowski norm $\left|\mathrm{P}_{k}(b)\right|_{\mu}$ of $b$ 's four-momentum as seen by $k$ (see Section 5 for the definition of $|\cdot|_{\mu}$ ), except that the actual, rather than absolute, value of $\mathrm{m}_{k}(b)$ is used. Thus the theorem states that the two observers agree both on the Minkowski length of $\mathrm{P}(b)$, and on the sign they assign to $b$ 's relativistic mass. As illustrated in the diagram, the points $\mathrm{P}_{k}=\left(\mathrm{m}_{k}, \mathbf{p}_{k}\right)$ and $\mathrm{P}_{h}=\left(\mathrm{m}_{h}, \mathbf{p}_{h}\right)$ must, therefore, lie on the same component of the Minkowski sphere $M$, and since $b$ is faster for $k$ than for $h$ the corresponding four-momentum is closer to the horizontal, whence it is clear that $\mathrm{m}_{k}<\mathrm{m}_{h}$ and $\left|\mathbf{p}_{k}\right|<\left|\mathbf{p}_{h}\right|$. 


\section{Outline of the proof}

Let $\|\cdot\|$ be either of the norms $|\cdot|_{\mu}$ or $|\cdot|_{\tau}$, where the Minkowski norm $|\bar{x}|_{\mu}$ and time norm $|\bar{x}|_{\tau}$ of $\bar{x} \in Q^{4}$ are given by

$$
|\bar{x}|_{\mu}=\sqrt{\left|x_{1}^{2}-x_{2}^{2}-x_{3}^{2}-x_{4}^{2}\right|} \quad \text { and } \quad|\bar{x}|_{\tau}=\left|x_{1}\right| .
$$

Recall that a linear transformation $L: Q^{4} \rightarrow Q^{4}$ is called a Lorentz transformation if it preserves the Minkowski norm. We will say that a linear transformation is time-preserving if it preserves the time-norm. An expansion on $Q^{4}$ is a mapping $E_{q}: Q^{4} \rightarrow Q^{4}$ given by $E_{q}(\bar{x}):=q \cdot \bar{x}$, where $0<q \in Q$.

The proof requires four basic lemmas.

1. Given two observers $k$ and $h$ moving with finite (possibly zero) speed with respect to one another, we show (Lemma 2) that there exists a slowly moving massive inertial particle $a$ on whose speed they agree (doing so first requires us to show (Lemma 1) that, subject to certain speed restrictions, we have $\mathrm{m}_{h}(b)=0$ whenever $\left.\mathrm{m}_{k}(b)=0\right)$. According to AxSpd ${ }^{-}$, the two observers therefore agree on the particle's relativistic mass as well, and hence $\left\|\mathrm{P}_{k}(a)\right\|=\left\|\mathrm{P}_{h}(a)\right\|$.

2. To prove the main theorem, we consider a collision between $a$ and an arbitrary massive particle $b$, and use basic linear algebra to deduce both that $\left\|\mathrm{P}_{k}(b)\right\|=\left\|\mathrm{P}_{h}(b)\right\|$ and that $k$ and $h$ agree as to the sign of $b$ 's relativistic mass, where the choice of norm depends on whether we are assuming AxPh or AxAbsSim in addition to DYN. In order to do this, we first need to prove that the linear part of the worldview transformation can be written as a composition of a norm-preserving transformation $L$ and an expansion $E_{q}$ (Lemma 3), and show under what conditions two observers assign the same sign to a particle's relativistic mass (Lemma 4).

Lemma 1. Assume DYN $\backslash\left\{\mathrm{AxSpd}^{-}\right\}$, and suppose $k, h \in \mathrm{IOb}$ and $b \in \mathrm{Ip}$ satisfy $v_{k}(b)<\infty$, $v_{h}(b)<\infty, v_{k}(h)<\infty$, and $v_{h}(k)<\infty$. Then $\mathrm{m}_{k}(b)=0$ if and only if $\mathrm{m}_{h}(b)=0$.

Proof. Suppose $\mathrm{m}_{k}(b)=0$. We will prove that $\mathrm{m}_{h}(b)=0$ as well. The converse will follow by symmetry.

Axiom AxThEx ${ }^{-}$says we can choose an inertial particle $a$ such that $\mathrm{m}_{k}(a) \neq 0, v_{k}(a)<\infty$

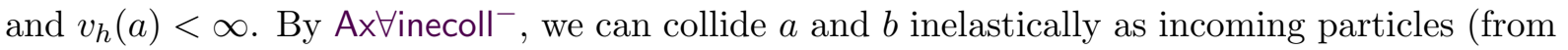
$k$ 's point of view) to form an outgoing particle $c$, and we have $\mathrm{P}_{k}(a)+\mathrm{P}_{k}(b)=\mathrm{P}_{k}(c)$. Because $\mathrm{m}_{k}(b)=0$, we know that $\mathrm{P}_{k}(b):=\left(\mathrm{m}_{k}(b), \mathrm{m}_{k}(b) \cdot \mathbf{v}_{k}(b)\right)=\overline{0}$, whence $\mathrm{P}_{k}(a)=\mathrm{P}_{k}(c)$ and $k$ considers $a$ and $c$ to have parallel worldlines. Axiom AxMass now tells us that $\mathrm{m}_{h}(a)=\mathrm{m}_{h}(c)$, and since $\mathbf{w}_{k h}$ is affine, it maps parallel worldlines to parallel worldlines, so $h$ considers $a$ and $c$ to have identical velocities. By definition, it follows that $\mathrm{P}_{h}(a)=\mathrm{P}_{h}(c)$.

We know that $a, b$ and $c$ form a collision for $k$, so they also form one for $h$ by $\mathrm{AxColl}_{3}$. We know that $a$ and $c$ have parallel worldlines from $k$ 's point of view, so, since they coincide at the collision point, the same straight line contains both of them in $k$ 's worldview, with the collision point marking a clear boundary between one worldline and the other. Since $\mathbf{w}_{k h}$ is affine, it takes straight lines to straight lines without changing the delimiting nature of the 'boundary point', so according to $h$, one of $a$ and $c$ is incoming and the other one is outgoing. Since they form a collision, we conclude that either

$$
\mathrm{P}_{h}(a)+\mathrm{P}_{h}(b)=\mathrm{P}_{h}(c) \quad \text { or } \quad \mathrm{P}_{h}(a)=\mathrm{P}_{h}(c)+\mathrm{P}_{h}(b)
$$

depending on whether $b$ is seen to be incoming or outgoing by $h$. In either case it follows that $\mathrm{P}_{h}(b)=\overline{0}$, whence $\mathrm{m}_{h}(b) \equiv\left(\mathrm{P}_{h}(b)\right)_{1}=0$ as claimed. 
The next lemma shows that two observers, moving at finite speed relative to one another, can identify a particle $b$ on whose speed they agree. The proof outline given below makes a simplifying assumption - equation (2) - and assumes that a version of Bolzano's theorem holds for $Q$ (i.e. if a continuous $Q$-valued function on $[0,1]$ takes both positive and negative values, then it must also take the value 0 at some point in the interval). A fully rigorous proof of the lemma is provided in the Appendix.

Lemma 2. Assume BA and $\mathrm{AxThEx}^{-}$, and suppose $k$ and $h$ are inertial observers satisfying $v_{k}(h)<\infty$ and $v_{h}(k)<\infty$. Then there exists an inertial particle $b$ such that $\mathbf{v}_{k}(b) \preceq \mathbf{v}_{k}(h)$, $v_{k}(b)=v_{h}(b)$ and $\mathrm{m}_{k}(b) \neq 0$.

Proof outline. If $v_{k}(h)=0$, then by AxThEx- , there is $b \in \mathrm{Ip}$ such that $v_{k}(b)=0$ and $\mathrm{m}_{k}(b) \neq 0$. This $b$ has the desired properties, so without loss of generality we shall assume that $v_{k}(h)>0$.

For simplicity assume that, for every inertial particle $b$,

$$
v_{k}(b) \leq v_{k}(h) \quad \Rightarrow \quad v_{h}(b)<\infty .
$$

This implication holds automatically if we assume either AxPh or AxAbsSim (see Corollary 1), as we do in the proof of the main theorem, but is not strictly necessary in the current context; see the Appendix.

For each $x \in[0,1]$, choose $b_{x} \in \operatorname{Ip}$ such that $\mathbf{v}_{k}\left(b_{x}\right)=x \cdot \mathbf{v}_{k}(h)$ and $\mathrm{m}_{k}\left(b_{x}\right) \neq 0$, and note that $b_{x}$ 's existence is guaranteed by AxThEx ${ }^{-}$. Since (2) implies that $v_{h}\left(b_{x}\right)<\infty$, we can define

$$
h(x)=v_{k}\left(b_{x}\right)-v_{h}\left(b_{x}\right)
$$

and it is easy to see that $h$ is continuous on $[0,1]$. By $v_{k}\left(b_{0}\right)=0, v_{h}\left(b_{0}\right)>0, v_{k}\left(b_{1}\right)>0$ and $v_{k}\left(b_{1}\right)=0$, we have that $h(0)<0$ and $h(1)>0$. Now Bolzano's theorem tells us there is some $u \in[0,1]$ such that $h(u)=0$, i.e. $v_{k}\left(b_{u}\right)=v_{h}\left(b_{u}\right)$. Taking $b=b_{u}$ completes the proof.

Lemma 3. Assume BA and suppose $k, h \in \mathrm{IOb}$. There exists a linear transformation $L$ and an expansion $E_{q}$ such that the linear part of $\mathbf{w}_{k h}$ is the composition $E_{q} \circ L$ of $L$ with $E_{q}$, viz.

$$
\mathbf{w}_{k h}(\bar{x})-\mathbf{w}_{k h}(\overline{0})=q \cdot L(\bar{x}) \quad \text { for all } \bar{x} \in Q^{4}, \quad \text { where }
$$

(a) if $\mathrm{AxPh}$ holds, $L$ is a Lorentz transformation;

(b) if AxAbsSim holds, $L$ is a time-preserving transformation.

Proof. (a) Notice first that $\mathbf{w}_{k h}$ is necessarily 'photon preserving', in that it maps any line of slope one in $k$ 's worldview to a line of slope one in $h$ 's worldview. To see why, suppose $\ell_{k}$ is such a line. By AxPh we can choose a photon $p$ whose worldline contains two points within $\ell_{k}$. Writing $\ell_{h}:=\mathbf{w}_{k h}\left(\ell_{k}\right)$, we know that $\ell_{h}$ is again a straight line (since $\mathbf{w}_{k h}$ is assumed affine, by AxW). Since $p$ is a photon and $\ell_{h}$ contains two points at which $p$ is present from $h$ 's point of view, it follows that the slope of $\ell_{h}$ is also equal to 1 (again by AxPh). But in [2, Theorem 3.6.4] it is shown that any photon-preserving affine transformation can be written as the composition of a Poincaré transformation and an expansion, and the result follows.

(b) Notice first that the linear part (call it $\Phi$ ) of $\mathbf{w}_{k h}$ maps the hyperplane $x_{1}=0$ to itself, since $\Phi(0, \bar{s})$ has to be simultaneous with $\Phi(\overline{0})=\overline{0}$ for all $\bar{s} \in Q^{3}$ by AxAbsSim. Since $\Phi$ is linear, it therefore maps the hyperplane $x_{1}=1$ to some hyperplane $x_{1}=\lambda$, where $\lambda \in Q$ is non-zero. It follows that $\Phi$ 's action can be written in the form $\Phi\left(x_{1}, \bar{s}\right)=\left(\lambda \cdot x_{1}, \phi(\bar{s})\right)$, where $\phi$ is a linear map on $Q^{3}$. Defining $q=|\lambda|$ ensures that $q>0$, so that the expansions $E_{q}$ and $E_{1 / q}$ are defined. If we now define $L=E_{1 / q} \circ \Phi$, it follows immediately that $L$ is time-preserving, and since $\Phi=E_{q} \circ\left(E_{1 / q} \circ \Phi\right)=E_{q} \circ L$, we are done. 
An easy corollary (since every inertial observer $h$ considers that $v_{h}(h)=0$ by AxSelf ${ }^{-}$) is that there are no FTL inertial observers in the relativistic case and no inertial observers moving with infinite speed in the Newtonian case.

Corollary 1. Assume BA and suppose $k, h \in \mathrm{IOb}$. Then $v_{k}(h)<1$ if $\mathrm{AxPh}$ holds, and $v_{k}(h)<\infty$ if AxAbsSim holds.

Given any inertial observer $k$ and incoming or outgoing inertial particle $b$, write

$$
\mathrm{A}_{k}(b)= \begin{cases}\mathrm{P}_{k}(b) & \text { if } b \text { is incoming } \\ -\mathrm{P}_{k}(b) & \text { if } b \text { is outgoing. }\end{cases}
$$

Lemma 4. Assume BA. Let $k$ and $h$ be inertial observers moving at finite speed relative to one another, and let $b$ be an inertial particle which $k$ considers to be either incoming or outgoing at some point and that $v_{h}(b)<\infty$. Suppose also that the linear part of $\mathbf{w}_{k h}$ is a composition $E_{q} \circ L$, where $E_{q}$ is an expansion $(q>0)$ and $L$ is a linear transformation.

Then

(a) if $\mathrm{A}_{h}(b)=L\left(\mathrm{~A}_{k}(b)\right)$, then $\mathrm{m}_{k}(b) \cdot \mathrm{m}_{h}(b) \geq 0$;

(b) if $\mathrm{A}_{h}(b)=-L\left(\mathrm{~A}_{k}(b)\right)$, then $\mathrm{m}_{k}(b) \cdot \mathrm{m}_{h}(b) \leq 0$.

Proof. Assume without loss of generality that $b$ is incoming for $k$. Then $\mathrm{A}_{k}(b)=\mathrm{P}_{k}(b)$. The proof when $b$ is outgoing is analogous. Moreover, if $\mathrm{m}_{k}(b)=0$, the claims follow trivially, so assume without loss of generality that $\mathrm{m}_{k}(b)>0$.

(a) If $b$ is incoming for $h$, then $\mathrm{A}_{h}(b)=\mathrm{P}_{h}(b)$ and so $\mathrm{P}_{h}(b)=L\left(\mathrm{P}_{k}(b)\right)$. Since $b$ is incoming for $h$ we know that its worldline exists to the past of the collision point, and likewise for $k$, so $L$ cannot have reversed the temporal sense of $b$ 's worldline, and hence cannot have changed the sign of the first component of $\mathrm{P}_{k}(b)$. On the other hand, if $b$ is outgoing for $h$, then $\mathrm{A}_{h}(b)=-\mathrm{P}_{h}(b)$ and so $\mathrm{P}_{h}(b)=-L\left(\mathrm{P}_{k}(b)\right)$. This time we know that $L$ must have reversed the temporal sense of $b$ 's worldline, since it maps a worldline to the past of the collision event to one that exists in its future. This is then reversed again by the negative sign in " $-L\left(\mathrm{P}_{k}(b)\right)$ ". In either case, we therefore find that the time-components of $\mathrm{P}_{k}(b)$ and $\mathrm{P}_{h}(b)$ must have the same sign (see Fig. 7). Since this component is the relativistic mass, we must have $\mathrm{m}_{h}(b)>0$, and the result follows.

(b) The proof of this part is similar. If $b$ is incoming for $h$, then $L$ does not reverse the temporal sense of $b$ 's worldline, but nonetheless $\mathrm{P}_{h}(b)=-L\left(\mathrm{P}_{k}(b)\right)$. On the other hand, if $b$ is outgoing for $h$, then $\mathrm{P}_{h}(b)=L\left(\mathrm{P}_{k}(b)\right)$ and $L$ has 'reversed time' (it has switched an incoming particle to an outgoing one). In either case, we find that the time-components of $\mathrm{P}_{k}(b)$ and $\mathrm{P}_{h}(b)$ must have opposite signs, and the result again follows.

Proof of main theorem. Suppose $k$ and $h$ are inertial observers and recall that, by Corollary 1, all inertial observers move at finite speed relative to one another, and that by Lemma 2, there exists a massive slow inertial particle $a$ on whose speed they agree.

Let $b$ be an arbitrary inertial particle. We will prove the theorem holds for $b$. Form a collision between $a$ and $b$, and call the resulting outgoing particle $c$. Without loss of generality we can assume that $c$ has finite speed relative to both $k$ and $h$, for if not we can use Ax $\forall$ inecoll ${ }^{-}$to replace $a$ with multiple copies of itself to change the resulting trajectory of $c$. Then by AxColl 3 we have that $a, b, c$ form a collision according to $h$. There are three cases to consider.

(a) If $\mathrm{m}_{k}(b)=0$, then we know from Lemma 1 that $\mathrm{m}_{h}(b)=0$, and so the theorem holds.

(b) Suppose $\mathbf{m}_{k}(b) \neq 0$. If $\mathbf{v}_{k}(b)=\mathbf{v}_{k}(a)$, then because $\mathbf{w}_{k h}$ is affine, we also have $\mathbf{v}_{h}(b)=$ $\mathbf{v}_{h}(a)$ and hence, by choice of $a$, that $v_{k}(b)=v_{h}(b)$. By AxSpd ${ }^{-}$it now follows that $\mathrm{m}_{k}(b)=$ 

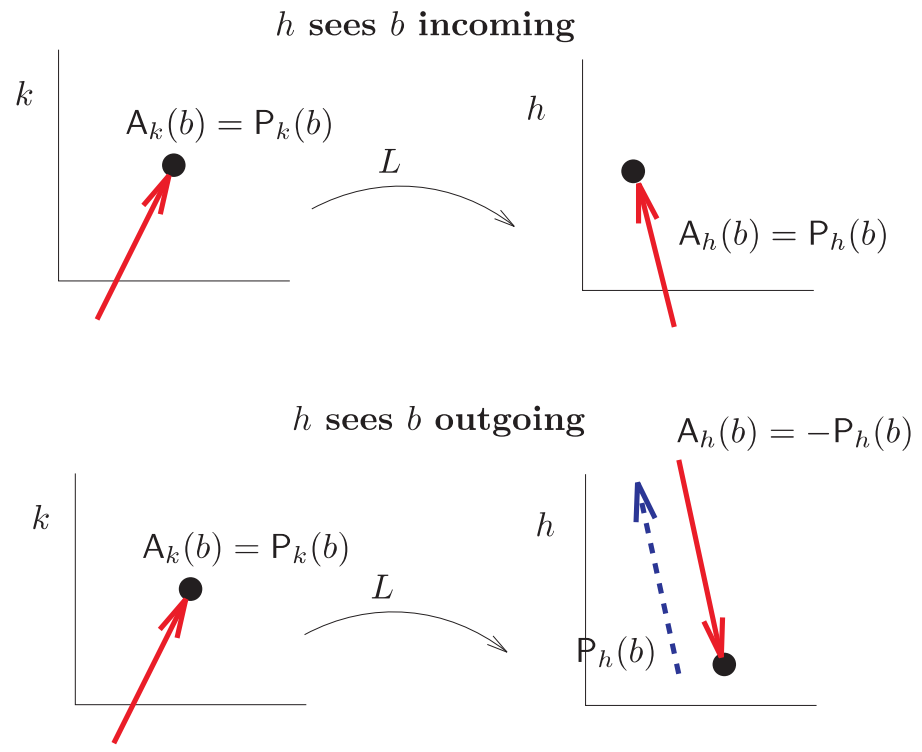

Figure 7. Illustration for the proof of (a) of Lemma 4.

$\mathrm{m}_{h}(b)$. So $k$ and $h$ agree on both $b$ 's speed and its relativistic mass, and the theorem again holds.

(c) Suppose $\mathrm{m}_{k}(b) \neq 0$ and $\mathbf{v}_{k}(b) \neq \mathbf{v}_{k}(a)$ (Fig. 8). Then the worldlines of $a$ and $b$ are not parallel in $k$ 's worldview, so their four-momenta cannot be linearly dependent. We also know that $\mathrm{P}_{k}(a)+\mathrm{P}_{k}(b)=\mathrm{P}_{k}(c)$ because $a, b$ and $c$ form a collision. It follows that no two of the vectors $L\left(\mathrm{P}_{k}(a)\right), L\left(\mathrm{P}_{k}(b)\right)$ and $L\left(\mathrm{P}_{k}(c)\right)$ can be linearly dependent.

Using the notation of Lemma 4 , the collision conditions on $a, b$ and $c$ can be written

$$
\mathrm{A}_{k}(a)+\mathrm{A}_{k}(b)+\mathrm{A}_{k}(c)=\overline{0}=\mathrm{A}_{h}(a)+\mathrm{A}_{h}(b)+\mathrm{A}_{h}(c)
$$

and hence

$$
\left(\mathrm{A}_{h}(a)-L\left(\mathrm{~A}_{k}(a)\right)\right)+\left(\mathrm{A}_{h}(b)-L\left(\mathrm{~A}_{k}(b)\right)\right)+\left(\mathrm{A}_{h}(c)-L\left(\mathrm{~A}_{k}(c)\right)\right)=\overline{0} .
$$

For each particle $d$, however, we know that $L\left(\mathrm{~A}_{k}(d)\right)$ is parallel to $\mathrm{A}_{h}(d)$, because each of these is parallel to $d$ 's worldline as seen by $h$. We claim that $\mathrm{A}_{h}(a)=L\left(\mathrm{~A}_{k}(a)\right)$. We have already observed that $L\left(\mathrm{~A}_{k}(a)\right)$ is parallel to $\mathrm{A}_{h}(a)$. To see that they have the same size (whether in the Minkowski norm, time norm or Euclidean norm), recall that (since $a$ is slow), $k$ and $h$ agree on $a$ 's speed, and also therefore (by $\mathrm{AxSpd}{ }^{-}$) on its relativistic mass. It now follows that $k$ and $h$ agree on the size of $\mathrm{A}(a)$, and since $L$ is norm-preserving $\mathrm{A}_{h}(a)$ and $L\left(\mathrm{~A}_{k}(a)\right)$ have the same size. It only remains to show that $\mathrm{A}_{h}(a)$ and $L\left(\mathrm{~A}_{k}(a)\right)$ point in the same direction. But this is immediate from Lemma $4(\mathrm{~b})$, since we would otherwise require $\mathrm{m}_{k}(a) \cdot \mathrm{m}_{h}(a) \leq 0$, which is impossible (we have already established that they are equal and non-zero).

It now follows from $(3)$ that $\left(\mathrm{A}_{h}(b)-L\left(\mathrm{~A}_{k}(b)\right)\right)+\left(\mathrm{A}_{h}(c)-L\left(\mathrm{~A}_{k}(c)\right)\right)=\overline{0}$, and hence by linear independence that

$$
\mathrm{A}_{h}(b)=L\left(\mathrm{~A}_{k}(b)\right) \quad \text { and } \quad \mathrm{A}_{h}(c)=L\left(\mathrm{~A}_{k}(c)\right) .
$$

Lemma 4(a) now tells us that $\mathrm{m}_{k}(b) \cdot \mathrm{m}_{h}(b) \geq 0$, while Lemma 1 shows that the inequality is strict, as claimed.

In summary, we know that $k$ and $h$ agree on the sign of the relativistic mass of $b$, and because $L$ is norm preserving, we know that $\left\|\mathrm{P}_{k}(b)\right\|=\left\|\mathrm{P}_{h}(b)\right\|$. For the relativistic case we 

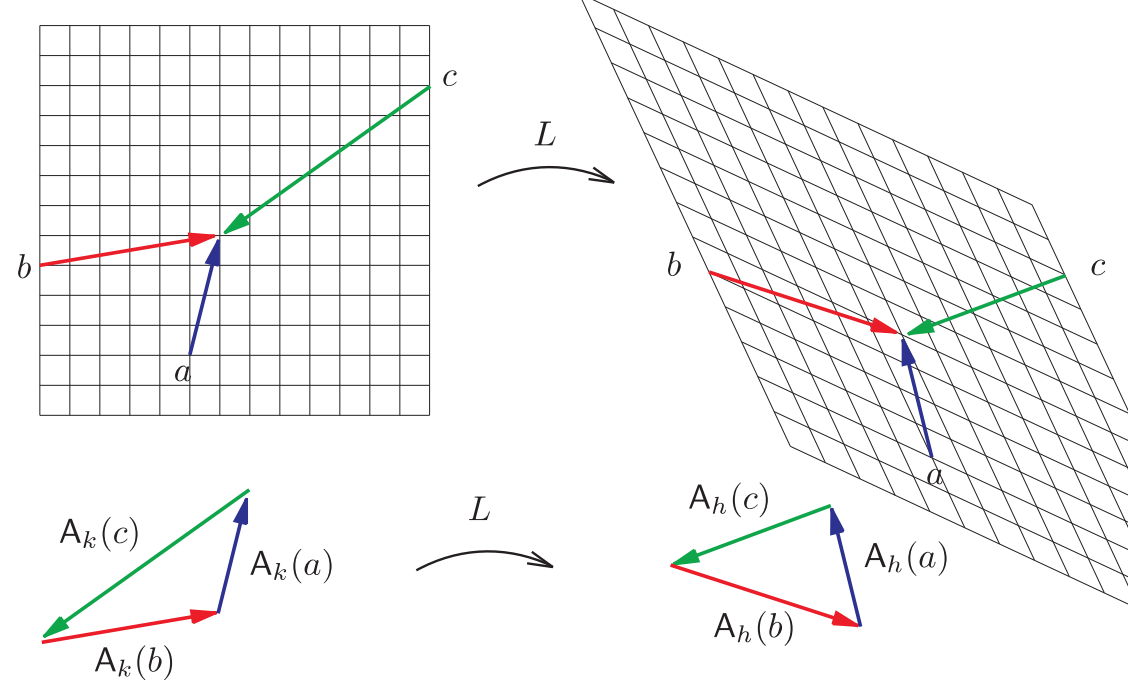

$$
\begin{array}{ll}
\mathrm{A}_{k}(a)+\mathrm{A}_{k}(b)+\mathrm{A}_{k}(c)=\overline{0} & \mathrm{~A}_{h}(a)+\mathrm{A}_{h}(b)+\mathrm{A}_{h}(c)=\overline{0} \\
v_{k}(a)=v_{h}(a) \wedge \mathrm{m}_{k}(a)=\mathrm{m}_{h}(a) \Rightarrow & L\left(\mathrm{~A}_{k}(a)\right)=\mathrm{A}_{h}(a) \Rightarrow \\
\Rightarrow L\left(\mathrm{~A}_{k}(b)\right)=\mathrm{A}_{h}(b) \text { and } L\left(\mathrm{~A}_{k}(c)\right)=\mathrm{A}_{h}(c) &
\end{array}
$$

Figure 8. Illustration for the proof of the main theorem.

have $\left\|\mathrm{P}_{k}(b)\right\|=\left|\mathrm{m}_{k}(b) \cdot\left(1, \mathbf{v}_{k}(b)\right)\right|_{\mu}=\left|\mathrm{m}_{k}(b)\right| \cdot \sqrt{\left|1-v_{k}^{2}(b)\right|}$, and likewise for $h$. Because $\mathrm{m}_{k}(b)$ and $\mathrm{m}_{h}(b)$ have the same sign, we can remove the relevant modulus signs, and conclude that

$$
\mathrm{m}_{k}(b) \cdot \sqrt{\left|1-v_{k}^{2}(b)\right|}=\mathrm{m}_{h}(b) \cdot \sqrt{\left|1-v_{h}^{2}(b)\right|} .
$$

In the Newtonian case, we have $\left\|\mathrm{P}_{k}(b)\right\|=\left|\left(\mathrm{m}_{k}(b), \mathrm{m}_{k}(b) \cdot \mathbf{v}_{k}(b)\right)\right|_{\tau}=\left|\mathrm{m}_{k}(b)\right|$, and hence

$$
\mathrm{m}_{k}(b)=\mathrm{m}_{h}(b)
$$

as claimed.

\section{Concluding remarks}

We began this paper by asking why the masses and momenta of faster-than-light particles decrease with their velocities. In the sense of [32], Theorem 1 answers this question by explaining that the axioms described in DYN+AxPh leave no other options open to us. The fact that our axiom system is so sparse, and the axioms themselves so elementary, makes this answer difficult to challenge, and highlights the advantages of the axiomatic approach. If experimental evidence one day shows our conclusions to be physically invalid, this will necessarily point to the invalidity also of one of our axioms, thereby identifying profitable areas for future investigation.

Alternatively, one can see the results presented here as a comment on our underlying axiom system - the axioms are strong enough to allow familiar and meaningful results to be derived for slow particles, yet generous enough not to disallow the existence of FTL particles for which reference frame transformations preserve norms as they do for slow particles ${ }^{4}$.

\footnotetext{
${ }^{4}$ We are grateful to the anonymous referee for this observation.
} 


\section{A Rigorous proof of Lemma 2}

Bolzano's theorem does not hold in general in arbitrary Euclidean ordered fields, but it holds for quadratic functions by item (i) of Lemma 5 , and as we shall see, this is all we require.

Recall that $r$ is a root of a function $f: Q \rightarrow Q$ iff $f(r)=0$, and that $f: Q \rightarrow Q$ is a quadratic function iff there are $a, b, c \in Q$ such that $f(x)=a x^{2}+b x+c$ for every $x \in Q$.

Lemma 5. Assume AxEField. Then (i) and (ii) below hold.

(i) Let $f$ be a quadratic function. Assume $p, q \in Q$ are such that $p<q, f(p)>0$ and $f(q)<0$. Then $f$ has a root between $p$ and $q$, i.e. there is $r \in Q$ such that $f(r)=0$ and $p<r<q$.

(ii) Let $f: Q \rightarrow Q$ be a function such that there are quadratic functions $f_{1}, f_{2}$ and $s \in Q$

$$
f(x)= \begin{cases}f_{1}(x) & \text { if } x \leq s \\ f_{2}(x) & \text { if } x \geq s\end{cases}
$$

Assume $p, q \in Q$ are such that $p<q$ and $f(p)>0$ and $f(q)<0$. Then $f$ has a root between $p$ and $q$.

Proof. To prove item (i), assume $f(x)=a x^{2}+b x+c$ for every $x \in Q$ and $f(p)>0, f(q)<0$ and $p<q$. Without loss of generality we can assume that $a>0$. Then, for every $x \in Q$,

$$
f(x)=a\left(x+\frac{b}{2 a}\right)^{2}-\frac{b^{2}-4 a c}{4 a} .
$$

If $b^{2}-4 a c \leq 0$, then $f(x) \geq 0$ for every $x \in Q$. Thus the discriminant $b^{2}-4 a c$ is positive since $f(q)<0$. Then, by the same methods as for the field of reals, one can prove that $f$ has exactly two roots $x_{1}, x_{2} \in Q$ such that $x_{1}<x_{2}$ and

$$
f(x)=a\left(x-x_{1}\right)\left(x-x_{2}\right) \quad \text { for every } x \in Q .
$$

Then $f(x) \leq 0$ if $x \in\left[x_{1}, x_{2}\right]$ and $f(x)>0$ if $x<x_{1}$ or $x>x_{2}$. By this, we conclude that $x_{1} \in[p, q]$, and this completes the proof of (i).

To prove item (ii) assume $f, f_{1}, f_{2}, s, p, q$ satisfy the assumptions. Let us note that $f_{1}(s)=f_{2}(s)$.

First assume that $s \leq p$. Then $f$ coincides with $f_{2}$ on $[p, q]$, i.e. $f(x)=f_{2}(x)$ for every $x \in[p, q]$. Then $f_{2}$ has a root between $p$ and $q$ by item (i) of the lemma. Hence $f$ has a root between $p$ and $q$.

Now assume that $s \geq q$. Then $f$ coincides with $f_{1}$ on $[p, q]$. Then by item (i) of the lemma we conclude that $f$ has a root between $p$ and $q$.

Finally assume that $p<s<q$. Then $f$ coincides with $f_{1}$ on $[p, s]$ and $f$ coincides with $f_{2}$ on $[s, q]$. If $f(s)<0$, then $f_{1}$ has a root between $p$ and $s$, and if $f(s)>0$ then $f_{2}$ has a root between $s$ and $q$ by item (i) of the lemma. Therefore, $f$ has a root between $p$ and $q$.

Proof of Lemma 2. Assume $k, h$ satisfy the assumptions. Let $L$ be the linear part of the world-view transformation $\mathbf{w}_{k h}$. In the proof we will need the following definition. Let $\bar{x} \in Q^{n}$ be such that $x_{1} \neq 0$. Then the speed $v(\bar{x})$ of vector $\bar{x}$ and line $(\bar{x})$ determined by vector $\bar{x}$ are defined as

$$
v(\bar{x}):=\frac{\sqrt{x_{2}^{2}+\cdots+x_{n}^{2}}}{\left|x_{1}\right|}, \quad \text { and } \quad \operatorname{line}(\bar{x}):=\{q \cdot \bar{x}: q \in Q\} .
$$




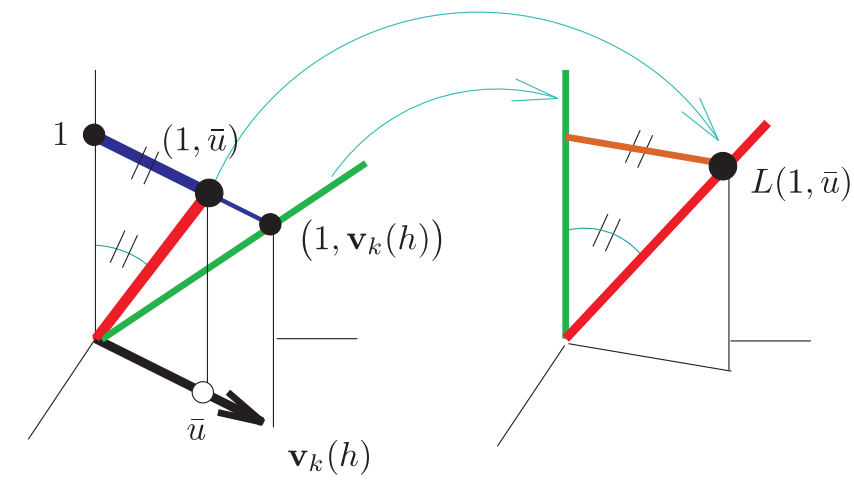

Figure 9. Illustration for the claim that (4) can be satisfied.

For every $\bar{u}, \bar{v} \in Q^{3}$, let

$$
\bar{u} \prec \bar{v} \stackrel{\text { def }}{\Longleftrightarrow} \bar{u} \neq \bar{v} \wedge \bar{u} \preceq \bar{v} .
$$

Claim. Assume $v_{k}(h)>0$. Then there is $\bar{u} \in Q^{3}$ such that

$$
\bar{u} \prec \mathbf{v}_{k}(h) \quad \text { and } \quad v(1, \bar{u})=v(L(1, \bar{u})),
$$

see Fig. 9.

We will prove the claim at the end of the proof. We note that in the field of reals, by continuity and Bolzano's theorem, it is easy to see that the claim holds.

If $v_{k}(h)=0$, then by AxThEx ${ }^{-}$there is an inertial particle $b$ such that $v_{k}(b)=0$ and $\mathrm{m}_{k}(b) \neq 0$. Then $v_{h}(b)=0$ and $b$ has the desired properties. Suppose instead, therefore, that $v_{k}(h)>0$. Then $v_{h}(k)>0$. Let $\bar{u} \in Q^{3}$ be such that (4) holds. Then, by AxThEx-, there is an inertial particle $b$ such that $\mathbf{v}_{k}(b)=\bar{u}$ and $\mathrm{m}_{k}(b) \neq 0$. Then $w \ell_{k}(b)$ is parallel with line $(1, \bar{u})$ and $w \ell_{h}(b)$ is parallel with line $(L(1, \bar{u}))$. Thus $v_{k}(b)=v(1, \bar{u})$ and $v_{h}(b)=v(L(1, \bar{u}))$. Thus $v_{k}(b)=v_{h}(b)$ and $\mathbf{v}_{k}(b) \prec \mathbf{v}_{k}(h)$.

Now we turn to prove the claim. Let $K: Q^{2} \rightarrow Q^{4}$ and $H: Q^{2} \rightarrow Q^{4}$ be the linear embeddings such that

$$
\begin{aligned}
& K(1,0)=(1,0,0,0) \quad \text { and } \quad K\left(0, v_{k}(h)\right)=\left(0, \mathbf{v}_{k}(h)\right), \quad \text { and } \\
& H(1,0)=(1,0,0,0) \quad \text { and } \quad H\left(0, v_{h}(k)\right)=\left(0, \mathbf{v}_{h}(k)\right) \text {. }
\end{aligned}
$$

Both $K$ and $H$ preserve the Euclidean distance and take the time-axis line $(1,0)$ of $Q^{2}$ to the time axis t-axis $:=\left\{\bar{x} \in Q^{4}: x_{2}=x_{3}=x_{4}=0\right\}$ of $Q^{4}$. Furthermore, for every $(t, x) \in Q^{2}$,

$$
v(t, x)=v(K(t, x))=v(H(t, x)) .
$$

Let

$$
T:=H^{-1} \circ L \circ K
$$

By BA, the world-view transformation $\mathbf{w}_{k h}$ takes $\mathrm{t}$-axis and $w \ell_{k}(h)$ to $w \ell_{h}(k)$ and $\mathrm{t}$-axis, respectively. Moreover, line $\left(1, \mathbf{v}_{k}(h)\right)$ and line $\left(1, \mathbf{v}_{h}(k)\right)$ are parallel with $w \ell_{k}(h)$ and $w \ell_{h}(k)$, respectively. Therefore,

$L$ takes t-axis to line $\left(1, \mathbf{v}_{h}(k)\right)$ and takes line $\left(1, \mathbf{v}_{k}(h)\right)$ to t-axis,

$K$ takes line(1,0) to t-axis and takes line $\left(1, v_{k}(h)\right)$ to line $\left(1, \mathbf{v}_{k}(h)\right)$,

$H$ takes line $(1,0)$ to $\mathrm{t}$-axis, and takes line $\left(1, v_{h}(k)\right)$ to line $\left(1, \mathbf{v}_{h}(k)\right)$. 
Therefore,

$T$ takes line $(1,0)$ to line $\left(1, v_{h}(k)\right)$,

$T$ takes line $\left(1, v_{k}(h)\right)$ to line $(1,0)$,

and $T: Q^{2} \rightarrow Q^{2}$ is a linear transformation.

Next we prove that

$$
\exists u\left[0<u<v_{k}(h) \wedge v(1, u)=v(T(1, u))\right] .
$$

Let $a, b, c, d \in Q$ be such that $T(t, x)=(a t+b x, c t+d x)$ for every $t, x \in Q$. Such $a, b, c, d$ exist and $a d-b c \neq 0$ since $T$ is a linear transformation. By (7), $T\left(1, v_{k}(h)\right)_{2}=0$. Thus $c+d \cdot v_{k}(h)=0$. Therefore, $c=-d \cdot v_{k}(h)$ and, for every $x \in Q, T(1, x)=\left(a+b x,-d \cdot v_{k}(h)+d x\right)$ and

$$
v(T(1, x))=\frac{|d| \cdot\left(v_{k}(h)-x\right)}{|a+b x|} \quad \text { if } a+b x \neq 0 \text { and } 0 \leq x \leq v_{k}(h) .
$$

By $c=-d \cdot v_{k}(h)$ and $a d-b c \neq 0$ we have that

$$
d \neq 0 \quad \text { and } \quad a+b \cdot v_{k}(b) \neq 0 .
$$

Clearly, $v(1, x)=x$ for every $x \geq 0$. Thus, to prove (8), we have to prove that

$$
\exists u\left[a+b u \neq 0 \wedge 0<u<v_{k}(h) \wedge \frac{|d| \cdot\left(v_{k}(h)-u\right)}{|a+b u|}=u\right] .
$$

Let us define a function $g: Q \rightarrow Q$ as follows:

$$
g(x):=|d| \cdot\left(v_{k}(h)-x\right)-|a+b x| \cdot x .
$$

If $u$ is a root of $g$, then by (9), $a+b u \neq 0$. Thus, we can prove (10) by showing that function $g$ has a root between 0 and $v_{k}(h)$.

Clearly, $g(0)=|d| \cdot v_{k}(h)$ and $g\left(v_{k}(h)\right)=-\left|a+b \cdot v_{k}(h)\right| \cdot v_{k}(h)$ by (11). Thus, by (9),

$$
g(0)>0 \quad \text { and } \quad g\left(v_{k}(h)\right)<0 .
$$

By Bolzano's theorem and (12), it is easy to prove that $g$ has a root between 0 and $v_{k}(h)$ if we assume that our field is the field of real numbers. To prove this for arbitrary Euclidean ordered fields, let $g_{0}, g_{1}$ and $g_{2}$ be the following quadratic functions:

$$
\begin{aligned}
& g_{0}(x):=|d| \cdot\left(v_{k}(h)-x\right)-|a| \cdot x, \\
& g_{1}(x):=|d| \cdot\left(v_{k}(h)-x\right)-(a+b x) \cdot x, \quad \text { and } \\
& g_{2}(x):=|d| \cdot\left(v_{k}(h)-x\right)+(a+b x) \cdot x .
\end{aligned}
$$

Assume that $b=0$. Then, $g(x)=g_{0}(x)$ for every $x$. By Lemma 5(i) and (12), we conclude that $g$ has a root between 0 and $v_{k}(h)$.

Now, assume $b \neq 0$. Then $i, j$ can be chosen such that $\{i, j\}=\{1,2\}$ and

$$
g(x)= \begin{cases}g_{i}(x) & \text { if } x \leq-a / b \\ g_{j}(x) & \text { if } x \geq-a / b\end{cases}
$$

Now, by Lemma 5 (ii) and (12), $g$ has a root between 0 and $v_{k}(h)$.

We have proved that (8) holds.

Let $u$ be such that

$$
0<u<v_{k}(h) \wedge v(1, u)=v(T(1, u)) .
$$

Let $\bar{u} \in Q^{3}$ be such that $K(1, u)=(1, \bar{u})$. Then $\bar{u} \prec \mathbf{v}_{k}(h)$ by definition of $K$ and (13). Furthermore, by (5), (6) and (13), we have that $v(1, \bar{u})=v(K(1, u))=v(1, u)=v(T(1, u))=$ $v\left(H^{-1} \circ L \circ K(1, u)\right)=v\left(H^{-1} \circ L(1, \bar{u})\right)=v(L(1, \bar{u}))$. Thus $v(1, \bar{u})=v(L(1, \bar{u}))$. Thus the claim holds, and this completes the proof of the lemma. 


\section{Acknowledgements}

This research is supported under the Royal Society International Exchanges Scheme (ref. IE110369) and by the Hungarian Scientific Research Fund for basic research grants No. T81188 and No. PD84093, as well as by a Bolyai grant for J.X. Madarász.

\section{References}

[1] Aharonov Y., Erez N., Reznik B., Superoscillations and tunneling times, Phys. Rev. A 65 (2002), 052124, 5 pages, quant-ph/0110104.

[2] Andréka H., Madarász J.X., Németi I., On the logical structure of relativity theories, Research report, Alfréd Rényi Institute of Mathematics, Hungar. Acad. Sci., Budapest, 2002, available at http://www.math-inst. hu/pub/algebraic-logic/Contents.html.

[3] Andréka H., Madarász J.X., Németi I., Székely G., Axiomatizing relativistic dynamics without conservation postulates, Studia Logica 89 (2008), 163-186, arXiv:0801.4870.

[4] Arntzenius F., Causal paradoxes in special relativity, British J. Philos. Sci. 41 (1990), 223-243.

[5] Bilaniuk O.M.P., Deshpande V.K., Sudarshan E.C.G., "Meta" relativity, Amer. J. Phys. 30 (1962), 718-723.

[6] Chashchina O.I., Silagadze Z.K., Breaking the light speed barrier, Acta Phys. Polon. B 43 (2012), 19171952, arXiv:1112.4714.

[7] d'Inverno R., Introducing Einstein's relativity, Oxford University Press, New York, 1992.

[8] Feinberg G., Possibility of faster-than-light particles, Phys. Rev. 159 (1967), 1089-1105.

[9] Feynman R.P., The theory of positrons, Phys. Rev. 76 (1949), 749-759.

[10] Firk F.W.K., Introduction to relativistic collisions, arXiv:1011.1943.

[11] Geroch R., Faster than light?, in Advances in Lorentzian Geometry, AMS/IP Stud. Adv. Math., Vol. 49, Amer. Math. Soc., Providence, RI, 2011, 59-69, arXiv:1005.1614.

[12] Jentschura U.D., Wundt B.J., Neutrino helicity reversal and fundamental symmetries, arXiv:1206.6342.

[13] Kleppner D., Kolenkow R.J., An introduction to mechanics, Cambridge University Press, Cambridge, 2010.

[14] Longhi S., Laporta P., Belmonte M., Recami E., Measurement of superluminal optical tunneling times in double-barrier photonic band gaps, Phys. Rev. E 65 (2002), 046610, 6 pages, physics/0201013.

[15] Madarász J.X., Székely G., The existence of superluminal particles is consistent with relativistic dynamics, arXiv:1303.0399.

[16] Nikolić H., Causal paradoxes: a conflict between relativity and the arrow of time, Found. Phys. Lett. 19 (2006), 259-267, gr-qc/0403121.

[17] Nimtz G., Heitmann W., Superluminal photonic tunneling and quantum electronics, Progr. Quantum Electron. 21 (1997), 81-108.

[18] Olkhovsky V.S., Recami E., Jakiel J., Unified time analysis of photon and particle tunnelling, Phys. Rep. 398 (2004), 133-178.

[19] Peacock K.A., Would superluminal influences violate the principle of relativity?, arXiv:1301.0307.

[20] Ranfagni A., Fabeni P., Pazzi G.P., Mugnai D., Anomalous pulse delay in microwave propagation: a plausible connection to the tunneling time, Phys. Rev. E 48 (1993), 1453-1460.

[21] Recami E., Classical tachyons and possible applications, Riv. Nuovo Cimento 9 (1986), 1-178.

[22] Recami E., Tachyon kinematics and causality: a systematic thorough analysis of the tachyon causal paradoxes, Found. Phys. 17 (1987), 239-296.

[23] Recami E., Superluminal tunnelling through successive barriers: does QM predict infinite group-velocities?, J. Modern Opt. 51 (2004), 913-923.

[24] Recami E., A homage to E.C.G. Sudarshan: superluminal objects and waves (an updated overview of the relevant experiments), arXiv:0804.1502.

[25] Recami E., The Tolman-Regge antitelephone paradox: its solution by tachyon mechanics, Electron. J. Theor. Phys. 6 (2009), 8 pages.

[26] Recami E., Zamboni-Rached M., Dartora C.A., Localized X-shaped field generated by a superluminal electric charge, Phys. Rev. E 69 (2004), 027602, 4 pages. 
[27] Rindler W., Relativity. Special, general, and cosmological, 2nd ed., Oxford University Press, New York, 2006.

[28] Stannett M., Németi I., Using Isabelle/HOL to verify first-order relativity theory, J. Autom. Reasoning, to appear, arXiv:1211.6468.

[29] Steinberg A.M., Kwiat P.G., Chiao R.Y., Measurement of the single-photon tunneling time, Phys. Rev. Lett. 71 (1993), 708-711.

[30] Stückelberg E.C.G., Un nouveau modèle de l'électron ponctuel en théorie classique, Helv. Phys. Acta 14 (1941), 51-80.

[31] Sudarshan E.C.G., The theory of particles traveling faster than light. I, in Symposia on Theoretical Physics and Mathematics (Madras, India), Editor A. Ramakrishnan, Plenum Press, New York, 1970, 129-151.

[32] Székely G., On why-questions in physics, in The Vienna Circle in Hungary, Editors A. Máté, M. Rédei, F. Stadler, Springer-Verlag, Wien, 2011, 181-189, arXiv:1101.4281.

[33] Tolman R.C., The theory of the relativity of motion, University of California, Berkeley, 1917.

[34] Zamboni-Rached M., Recami E., Besieris I.M., Cherenkov radiation versus X-shaped localized waves, J. Opt. Soc. Amer. A 27 (2010), 928-934.

[35] Zamboni-Rached M., Recami E., Besieris I.M., Cherenkov radiation versus X-shaped localized waves: reply, J. Opt. Soc. Amer. A 29 (2012), 2536-2541. 